\title{
Repeated Nicotine Strengthens Gamma Oscillations in the Prefrontal Cortex and Improves Visual Attention
}

\author{
Lezio S Bueno-Junior',2, Nicholas W Simon', Meredyth A Wegener' and Bita Moghaddam*,1 \\ 'Department of Neuroscience, University of Pittsburgh, Pittsburgh, PA, USA; ${ }^{2}$ Ribeirão Preto Medical School, University of São Paulo, Ribeirão Preto, \\ Brazil
}

\begin{abstract}
Nicotine has strong addictive as well as procognitive properties. While a large body of research on nicotine continues to inform us about mechanisms related to its reinforcing effects, less is known about clinically relevant mechanisms that subserve its cognitive-enhancing properties. Understanding the latter is critical for developing optimal strategies for treating cognitive deficits. The primary brain region implicated in cognitive functions improved by nicotine is the prefrontal cortex (PFC). Here we assessed the impact of nicotine on unit activity and local field potential oscillations in the PFC of behaving rats. An acute dose of nicotine produced a predominantly inhibitory influence on population activity, a small increase in gamma oscillations, and a decrease in theta and beta oscillations. After a daily dosing regimen, a shift to excitatory-inhibitory balance in single-unit activity and stronger gamma oscillations began to emerge. This pattern of plasticity was specific to the gamma band as lower frequency oscillations were suppressed consistently across daily nicotine treatments. Gamma oscillations are associated with enhanced attentional capacity. Consistent with this mechanism, the repeat dosing regimen in a separate cohort of subjects led to improved performance in an attention task. These data suggest that procognitive effects of nicotine may involve development of enhanced gamma oscillatory activity and a shift to excitatory-inhibitory balance in PFC neural activity. In the context of the clinical use of nicotine and related agonists for treating cognitive deficits, these data suggest that daily dosing may be critical to allow for development of robust gamma oscillations.

Neuropsychopharmacology (2017) 42, 1590-1598; doi:I0.1038/npp.20 17.15; published online I March 2017
\end{abstract}

\section{INTRODUCTION}

There is a well-established dichotomy in the clinical effects of nicotine. On the one hand, it can cause chemical dependence with detrimental health consequences (Picciotto, 1998). On the other hand, it is a cognitive enhancer with potential for treating symptoms of major psychiatric disorders such as schizophrenia (Freedman, 2014). Extensive work continues to inform us about mechanisms underlying nicotine's reinforcing properties (De Biasi and Dani, 2011; Markou, 2008), but less is known about mechanisms that subserve nicotine's cognitive-enhancing effects. Understanding the latter is critical for developing nicotinic cognitive agents devoid of addictive properties.

Sustained attention performance, which relies on neuronal activity in medial prefrontal cortex (mPFC) subregions (Totah et al, 2009), is improved by repeated exposure to nicotine or selective nicotinic receptor agonists (Levin et al, 2006; McGaughy et al, 1999; Semenova et al, 2007). Attentional impairment is a hallmark of several psychiatric disorders including schizophrenia (Kahn and Keefe, 2013) and nicotinic

* Correspondence: Dr B Moghaddam, Department of Behavioral Neuroscience, Oregon Health and Science University, L470 3181, S.W. Sam Jackson Park Rd., Portland OR 97239, USA, Tel: 503402 2858, E-mail: bita@ohsu.edu

Received 15 July 2016; revised 14 December 2016; accepted 22 December 2016; accepted article preview online 27 January 2017 receptor activation has been suggested as a therapeutic option for these impairments (Freedman, 2014). Understanding how nicotine alters brain activity and behavior across multiple treatments is critical for determining an optimal therapeutic schedule that supports mPFC-mediated cognition.

The procognitive effects of nicotine may be related to neuronal ensemble modulation in the mPFC. Gamma band local field potentials (LFPs) reflect network synchrony as determined by the balance between excitatory and inhibitory activity within a region (Sohal et al, 2009; Uhlhaas and Singer, 2013). Gamma oscillations coordinated by fast-spiking interneurons (FSIs) in $\mathrm{mPFC}$ are required for attentional processing, and increased gamma oscillations improve attention (Kim et al, 2016). Additionally, aberrant gamma oscillations have been proposed as a biomarker for cognitive deficits in schizophrenia (Uhlhaas and Singer, 2013). This possibility is supported by reduced gamma band activity in the mPFC of individuals with schizophrenia during attention tasks (Ferrarelli et al, 2008). Nicotine affects gamma oscillations in hippocampus in slice (Wang et al, 2015); however, the effects of nicotine on mPFC gamma and other LFP frequency bands remain unclear.

Here, we investigated the impact of acute and repeated nicotine on mPFC neuronal activity and locomotion by recording LFP and single units in behaving animals. We discovered that repeated nicotine selectively strengthens gamma oscillations in correlation with behavioral sensitization. 
Because gamma oscillations are associated with enhanced attentional capacity, we performed a follow-up behavior study and found that repeated nicotine also strengthens attention. These data support the notion that the procognitive effects of nicotine may require extended exposure in order to produce oscillatory plasticity in the mPFC.

\section{MATERIALS AND METHODS}

\section{Subjects}

Adult male Long-Evans rats ( 85-90 postnatal days) were used and housed in reverse light/dark cycle (lights on at 1900 hours). Data collection was performed during the wake hours (dark cycle). All procedures were in accordance with the National Institute of Health's Guide for the Care and Use of Laboratory Animals, and were approved by the University of Pittsburgh's Institutional Animal Care and Use Committee.

\section{Nicotine Dosing}

Nicotine hydrogen tartrate salt (Sigma-Aldrich, USA) was dissolved in saline. An intraperitoneal (i.p.) dose of $0.2 \mathrm{mg} / \mathrm{kg}$ was administered for five consecutive days (Figure 1), followed by a nine-day washout period, and a final dose on day 10 (referred to as the 'late' session; Figure 1). This commonly used dose of nicotine was used because it influences brain oscillatory activity as measured by EEG (Ferger and Kuschinsky, 1994) and produces behavioral sensitization after repeated dosing (DiFranza and Wellman, 2007). This dose is less than the standard nicotine dose in a patch, greater than the amount in a single piece of gum, and comparable to half a pack of cigarettes, although the rate and success of absorption differs substantially between i.p. injections and these other routes of administration (Chang et al, 2016).

\section{Electrophysiology in Behaving Rats}

Electrophysiology and surgical methods were similar to that described before (Homayoun and Moghaddam, 2007; Wood et al, 2012). Electrodes (eight $50 \mu \mathrm{m}$ wires) were bilaterally implanted in the prelimbic region of the $\mathrm{mPFC}(+3.0 \mathrm{~mm}$ $\mathrm{AP}, \pm 0.6 \mathrm{~mm} \mathrm{ML},-3.2 \mathrm{~mm} \mathrm{DV})$ in 15 rats. Recording sessions began after at least five days of recovery. A unitygain headstage (Plexon, USA) connected to a motorized commutator was used to allow free movement (Figure 1). The electrophysiological signal was amplified and filtered for LFP $(0.7-300 \mathrm{~Hz}$ bandpass) and single-unit activity (150$8000 \mathrm{~Hz})$ via an OmniPlex acquisition system (Plexon). Spikes were sorted using Offline Sorter (Plexon). A recording session included a $30 \mathrm{~min}$ baseline, i.p. saline $+30 \mathrm{~min}$ postsaline, followed by i.p. nicotine $+60 \mathrm{~min}$ post-nicotine. Locomotor activity was tracked throughout. At the termination of each experiment, animals were anesthetized with chloral hydrate and perfused with saline and $10 \%$ formalin. Brains were sectioned and Nissl stained to confirm electrode placements.

\section{Visual Attention Task}

Visual attention performance (adapted from McGaughy and Sarter, 1995) in response to the same 5-day regimen of a
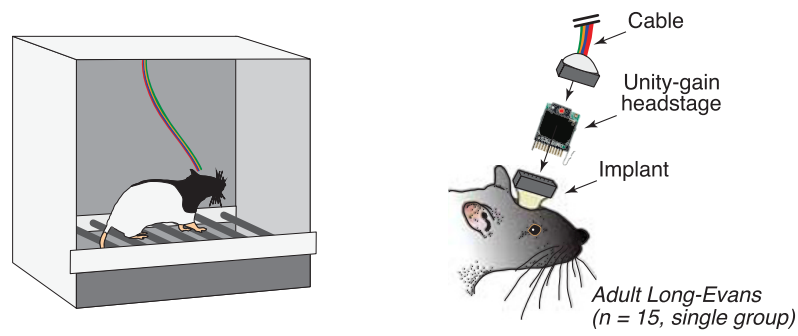

b
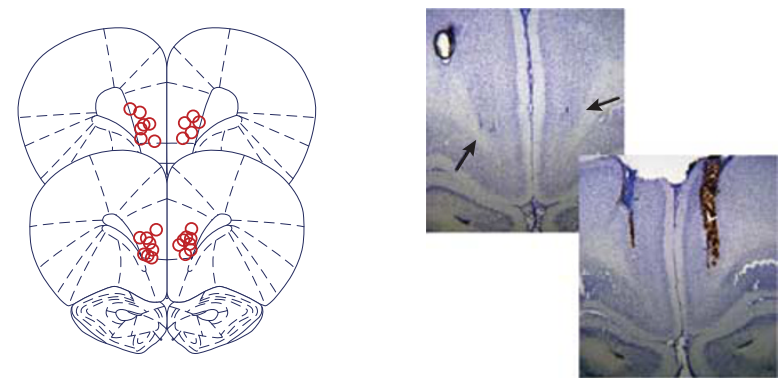

C

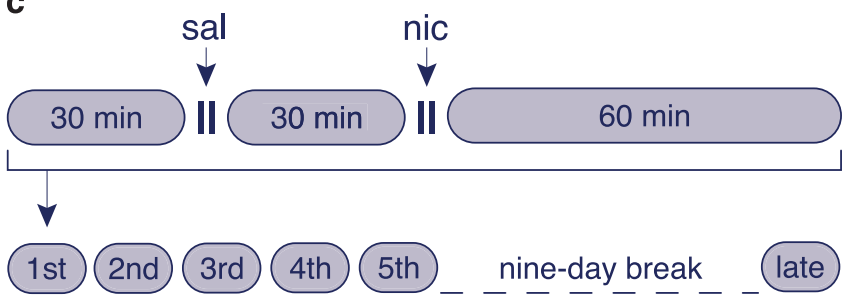

c
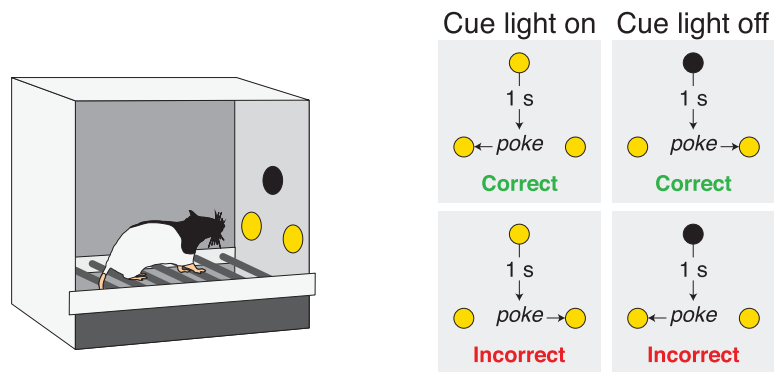

Figure I Experimental design and electrode placement. (a) General aspects of experimental design. (b) Histologically confirmed placements of microelectrode bundles in the medial prefrontal cortex (mPFC) (left) and Nissl-stained sections from two representative rats (right). (c) Timeline of individual electrophysiological sessions (top) and daily $0.2 \mathrm{mg} / \mathrm{kg}$ dose nicotine regimen (bottom). Recording during nicotine exposure was performed for five consecutive days, followed by a nicotine-free period and a post-withdrawal session (designated as 'late'). (d) Schematics of the visual attention experiment, adapted from a previous work (McGaughy and Sarter, 1995). In brief, in 50\% of trials, a centrally located cue light would illuminate $(25,50$ or $100 \mathrm{~ms})$. Then, after I s, both response ports were lit. A response on the left side was correct for this trial type, and was reinforced with a pellet. In the other $50 \%$ of trials, the cue was not illuminated prior to the ports being lit, and a response on the right side was reinforced. The test phase of such experiment was performed under the same nicotine regimen shown in the lower portion of panel (c). Each experiment employed a separate cohort of rats.

nicotine $(0.2 \mathrm{mg} / \mathrm{kg})$ was tested in a separate cohort. Rats were food restricted to $90 \%$ of free feeding weight. After habituation to the operant chamber $(30 \times 30 \times 25 \mathrm{~cm}$; Harvard Apparatus, USA) and fixed ratio 1 operant training, the 
visual attention task began with presentation of either a $1 \mathrm{~s}$ light cue or no cue and, after a 1 s delay, both ports were lit. A poke into the left port after a cued trial was rewarded with pellet delivery and food trough illumination, followed by an intertrial interval (ITI) of $12 \pm 3 \mathrm{~s}$. A poke into the right port after a cue was scored as an incorrect trial and triggered the ITI. In non-cued trials, a poke into the right port was reinforced with a pellet, while the left port triggered the ITI (Figure 1). Cue-side pairings were counterbalanced across subjects. Failing to respond within $3 \mathrm{~s}$ caused termination of port lights, and the trial was scored as an omission. After an incorrect response, that trial type was repeated until the correct port was selected. This assisted with acquisition and prevented side bias. Otherwise, trials were presented in random order. The number of trials completed during a 45-min session (approximately 180) varied depending on response rate.

Once rats achieved $70 \%$ correct trials in a session, they progressed to the full visual attention task. This task was similar to the training, except cue duration was reduced, and incorrect trials did not force repetition of the same trial type. There were four trial types: 100, 50, or $25 \mathrm{~ms}$ cue, and non-cued trials. The trials were presented in random order, with 50\% cued and 50\% non-cued. Attentional accuracy was assessed as the percentage of correct responses across all cued trials. Once an animal reached criterion (70\% correct at $100 \mathrm{~ms}$ cue trials only, across 2 days), it was assigned to the saline or nicotine group. Injections were made 5 min before sessions 1 through 5 , and once more before session 15 .

\section{Data Analysis}

Electrophysiology data were analyzed using custom MATLAB scripts (Mathworks, USA) similar to that described before (Wood et al, 2012). We focused our analysis on days 1, 3, 5, and the 'late' session. Briefly, LFPs were processed using the Chronux toolbox for MATLAB, www. chronux.org (Mitra and Bokil, 2008). To reduce variability in the data, spectral densities were estimated through the multitaper method (frequency pass: $0-140 \mathrm{~Hz}$; sliding time windows: $30 \mathrm{~s}, 2 \mathrm{~s}$ steps; time-bandwidth product: 3; tapers: 5). Using the resulting spectrogram arrays, frequency bins were Z-scored against their baselines. For each recording session (injection day), data from all rats were averaged and plotted as mean spectrograms. Frequency bands were averaged for theta-beta $(5-25 \mathrm{~Hz})$, low gamma $(40-60 \mathrm{~Hz})$, and high gamma $(60-130 \mathrm{~Hz})$ bands and then averaged across rats in 3 min bins. Two-way ANOVAs with time bins as repeated measures and Tukey's post hoc tests were used to compare between sessions, either 1st vs 5th or 1st vs late. One-way ANOVAs were used for within-session analyses.

For locomotion data, movement timestamps were tracked every $3 \mathrm{~min}$ and presented as mean \pm SEM movement counts. Similar to LFP analysis, statistical comparisons were made between (two-way ANOVA) and within (one-way ANOVA) sessions.

For single unit analysis, spike count histograms (1 min bins) were $\mathrm{Z}$-scored against their baselines and visualized as heat plots, providing overviews of $\mathrm{mPFC}$ single-unit activity. The results are shown only for sessions 1, 3, and 5, as the post-washout session yielded a low number of units. T-test was used for comparison between the baseline and postnicotine period. Single units were then categorized according to the post-nicotine activity: increased, decreased or unchanged. A $\chi^{2}$ test was used to evaluate if the proportions of these categories changed throughout sessions. Time binning was then converted to $3 \mathrm{~min}$, and two-way repeated measures ANOVAs and Tukey's post-hoc tests were used to compare between sessions. Only $2.5 \%$ of units were putative FSIs, as identified by a combination of high baseline firing rate $(>10 \mathrm{~Hz})$ and narrow spike width $(<0.3 \mathrm{~ms}$ between valley and peak; (Homayoun and Moghaddam, 2007)). Units meeting these criteria were removed from the main samples and separately analyzed.

For the visual attention task, the mean accuracy (percentage of correct responses) for nicotine and saline groups were assessed across sessions. We compared accuracy at each cue length using a two-way cue $v s$ session ANOVA, and also assessed overall accuracy on cued trials using a two-way drug $v s$ session ANOVA. Individual sessions were compared between groups using individual $t$-tests with Bonferroni's correction $(p \leqslant 0.01)$. Other behavioral measures assessed and compared between nicotine and saline groups were total omissions, premature responses, perseverative errors, and latency to respond.

\section{RESULTS}

\section{mPFC Gamma Oscillations Increase with Repeated Doses of Nicotine}

Figure 2 demonstrates that nicotine's effects on low gamma oscillations become more pronounced after multiple treatments (Figure 2). The first nicotine exposure increased low gamma power significantly relative to baseline $\left(\mathrm{F}_{(2,36)}=7.51\right.$, $p=0.002)$. This effect was larger on day $3\left(\mathrm{~F}_{(2,33)}=23.90\right.$, $p<0.001)$ and on day $5\left(\mathrm{~F}_{(2,30)}=12.70, p<0.001\right.$; Figure $\left.2 \mathrm{a}\right)$. There was a significant difference in low gamma across sessions in that gamma power on days 3 and 5 were greater than day $1\left(\mathrm{~F}_{(2,33)}=3.38, p<=0.04\right)$. There also was a greater potentiation of low gamma power in session 5 , as indicated by time $v s$ day interaction between sessions 1 and 5 $\left(\mathrm{F}_{(19,418)}=3.64, p<0.001\right.$; Figure $\left.2 \mathrm{~b}\right)$. Finally, within-session comparisons showed that low gamma is enhanced in the post-washout late session $\left(\mathrm{F}_{(2,24)}=27.33, p<0.001\right.$; Figure $2 \mathrm{a}$ and $b$ ) and that nicotine affects low gamma power more robustly in the late session compared with day 1 $\left(\mathrm{F}_{(1,380)}=7.54, p=0.01\right.$; Figure $\left.2 \mathrm{~b}\right)$.

Potentiation of high gamma by nicotine emerged after session 1 (session $1: p=0.1 ; 3$ and $5: p<0.02$ ) and was most strongly modulated on the post-washout late session (late session $v$ s day $1: \mathrm{F}_{(1,380)}=8.98, p=0.007$; Figure $\left.2 \mathrm{~b}\right)$. Nicotine also influenced lower (theta-beta) oscillations, but in contrast to the plasticity observed in gamma frequencies, this effect remained constant after repeated treatment (Fs $>3.60$, ps $<0.04$; Figure $2 \mathrm{a}$ and $\mathrm{b}$ ).

\section{Repeated Nicotine Produces Locomotor Sensitization in Correlation with Increased Gamma Power}

Locomotor behavior in response to nicotine was sensitized during electrophysiological recordings. Nicotine did not affect locomotion on days 1 or 3 (ps $>0.38$ ) but did so on 
a
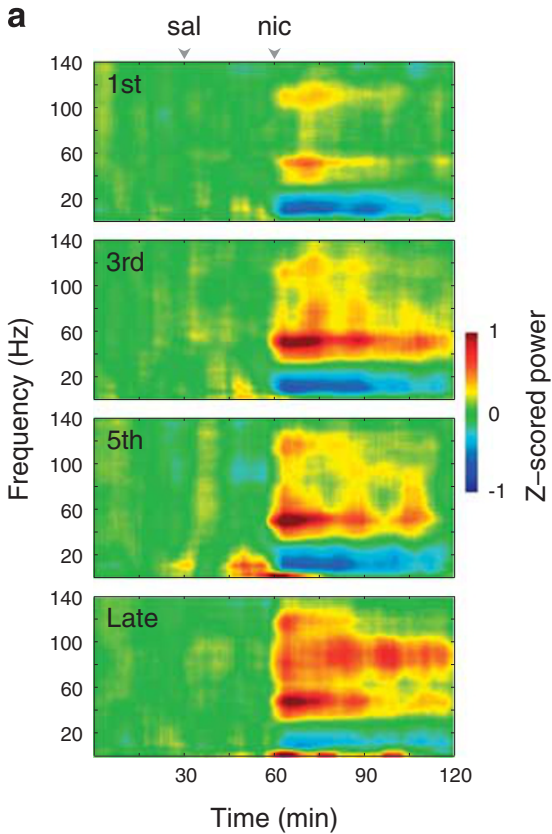

b
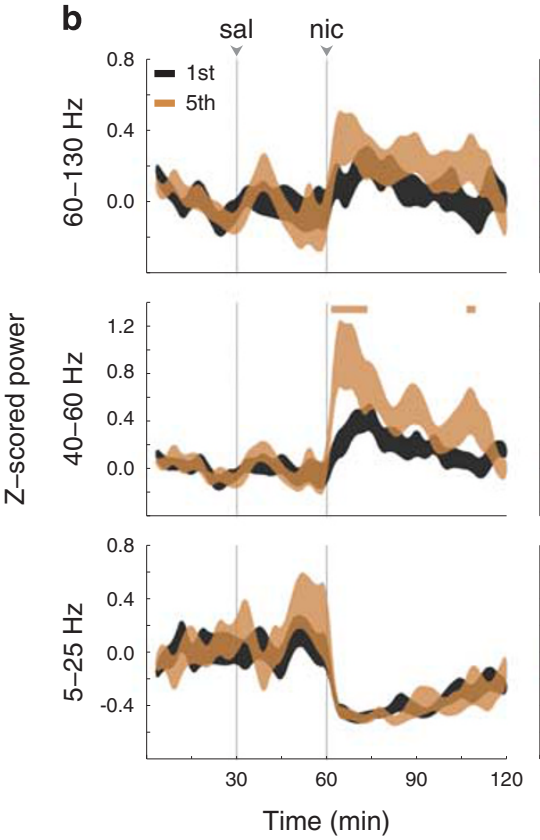
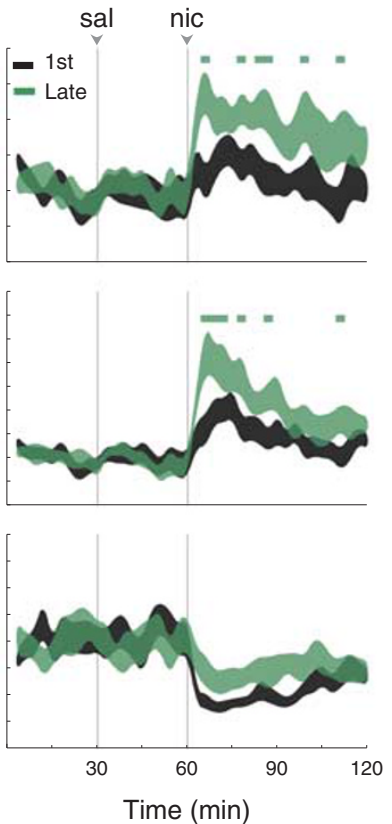

c

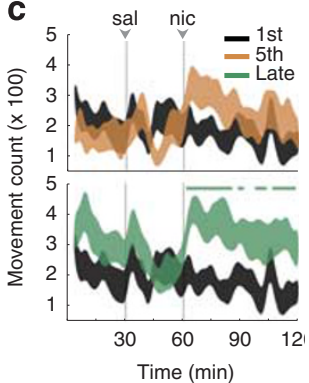

d

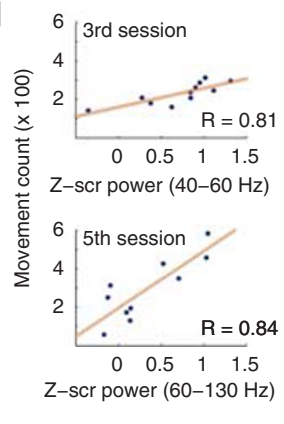

Figure 2 Nicotine potentiated medial prefrontal cortex (mPFC) gamma oscillations and locomotion upon daily exposures. (a) mPFC oscillatory activity throughout nicotine sessions. Each image is the average of Z-scored spectrograms from individual rats (color bar: Z-scored power). (b) From top to bottom, the graphs compare high-gamma, low-gamma, and theta-beta oscillations, either between days I and 5 (left) or between day I and the late session (right). Shaded curves represent \pm SEM, and Tukey's significant effects between days are indicated above each panel (comparing 3 min bins). (c) Comparison of movement counts either between days I and 5 (top) or day I and the late session (bottom). (d) Scatter plots correlating averaged data from the first I 5 min after nicotine administration. Top: movement count vs low gamma power in the third session. Bottom: movement count vs high gamma power in the fifth session. Each point represents a rat. Pearson's $R$ statistics showing significant correlations are also displayed.

day 5 (compared with baseline $\mathrm{F}_{(2,30)}=3.77, p=0.03$; compared with day $1, \mathrm{~F}_{(1,399)}=3.92, p=0.06$; Figure $\left.2 \mathrm{c}\right)$. A significant increase in locomotion was also observed in the late session (compared with baseline, $\mathrm{F}_{(2,27)}=5.89$, $p=0.008$; compared with day $1, \mathrm{~F}_{(1,380)}=13.63, p=0.001$; Figure $2 \mathrm{c}$ ). We also observed a significant positive correlation between locomotion and low gamma power on day 3 $(R=0.815, p=0.002)$, and between locomotion and high gamma power on day $5(R=0.84, p=0.002$; Figure $2 \mathrm{~d})$, suggesting a link between gamma oscillations and development of nicotine-induced locomotor sensitization (Table 1).

\section{Daily Nicotine Induced Changes in mPFC Single Unit Activity}

Nicotine changed spontaneous activity of mPFC units with a mixed pattern of activation and inhibition (Figure 3a). On day 1 , the majority of units were inhibited, whereas on days 3 and 5 , more units were excited with no cumulative inhibition or excitation (Figure 3b). Table 2 shows the proportions of units exhibiting each response pattern $\left(\chi^{2}=10.311 ; p=0.03\right)$, suggesting that nicotine's effects shift from net inhibition to an inhibition/excitation balance across daily treatments. Population activity of mPFC single units was significantly different between days 1 and 3 (effect of sessions: $\left.\mathrm{F}_{(1,2508)}=4.62, p=0.03\right)$, but not between 1 and 5, suggesting that a net excitation was confined to day 3 (Figure $3 \mathrm{c}$ ). Analyses of the absolute population activity (disregarding the direction of changes from baseline) show that nicotine modulation of population activity was consistent across sessions, although at a slightly higher latency from nicotine injection on day 5 (day 1 vs 3 effect of session
Table I Correlation Analyses Between Locomotion and Oscillation Power

\begin{tabular}{|c|c|c|c|c|c|c|c|c|}
\hline & \multicolumn{8}{|c|}{ Movement count } \\
\hline & \multicolumn{2}{|c|}{ Day I } & \multicolumn{2}{|c|}{ Day 3} & \multicolumn{2}{|c|}{ Day 5} & \multicolumn{2}{|c|}{ Late } \\
\hline & $R$ & P & $R$ & $p$ & $R$ & p & $R$ & p \\
\hline High gamma & -0.421 & 0.173 & 0.350 & 0.291 & 0.843 & 0.002 & -0.048 & 0.909 \\
\hline Low gamma & -0.375 & 0.230 & 0.815 & 0.002 & 0.563 & 0.090 & -0.296 & 0.477 \\
\hline Theta-beta & 0.009 & 0.979 & 0.256 & 0.447 & -0.278 & 0.437 & 0.666 & 0.071 \\
\hline
\end{tabular}

Significant correlations are in bold. Data were averaged from the initial 15 min after nicotine.

time: $\mathrm{F}_{(19,2508)}=7.63, p<0.001$; day 1 vs 5 effect of session time: $\mathrm{F}_{(19,2451)}=3.55, p<0.001$; day 1 vs 5 interaction: $\left.\mathrm{F}_{(19,2451)}=1.99, p=0.007\right)$. We could only record from 17 units at the late session and thus could not perform reliable statistical analyses with that data set.

A small sample of units $(n=5)$ were identified as putative FSIs (Figure 3d). While this subgroup is not large enough to justify conclusions, it is noteworthy that FSIs were excited by nicotine exposure (one-way repeated measures ANOVA: $\left.\mathrm{F}_{(39,156)}=2.45, p<0.001\right)$.

\section{Daily Nicotine Improved Visual Attention on the Same Timescale as Low Gamma Modulation}

For this experiment, nicotine dosing regimen was equivalent to the electrophysiology experiment (Figure 1). There was no 
a
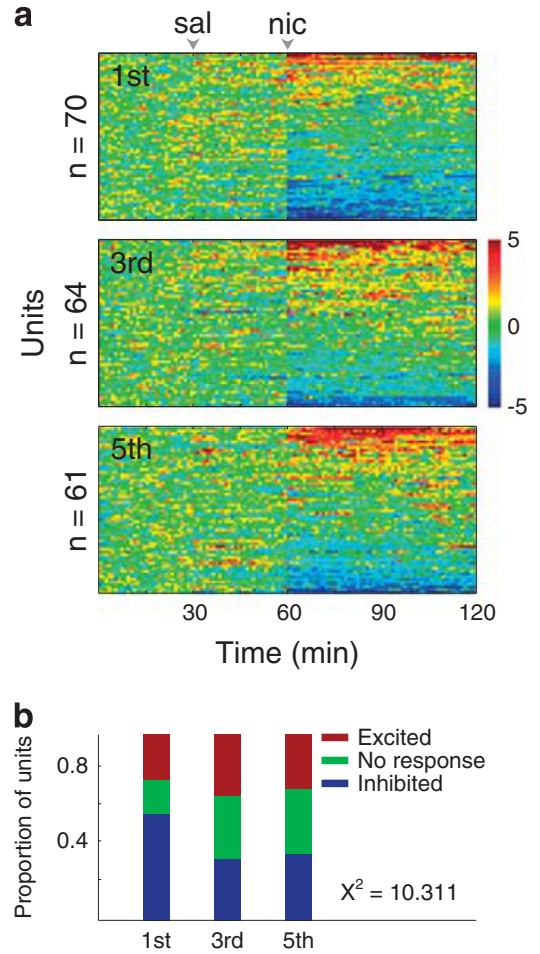

C
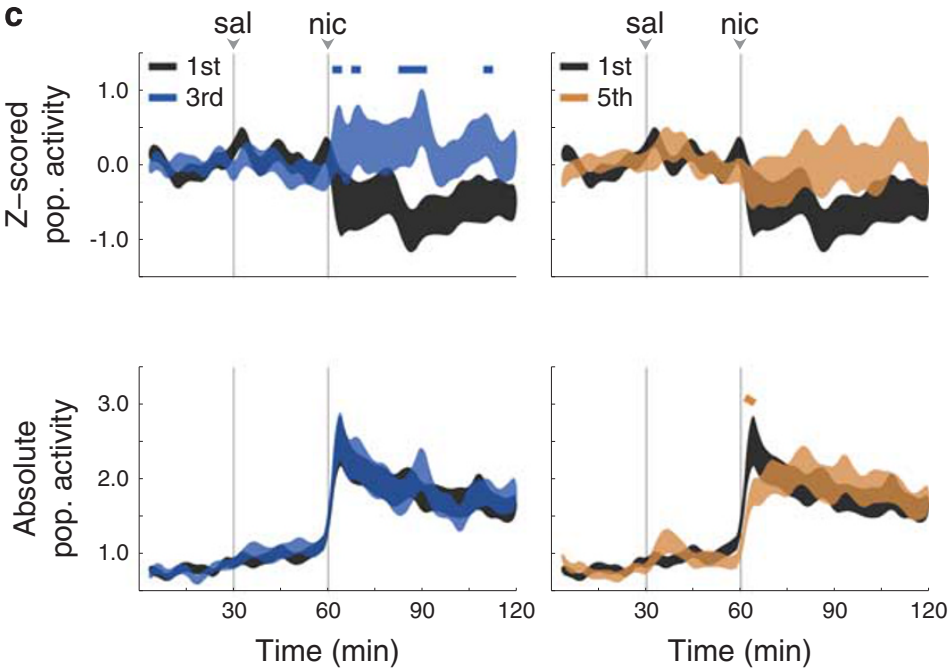

d

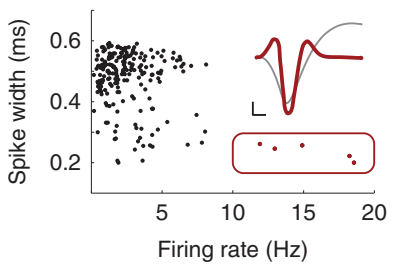

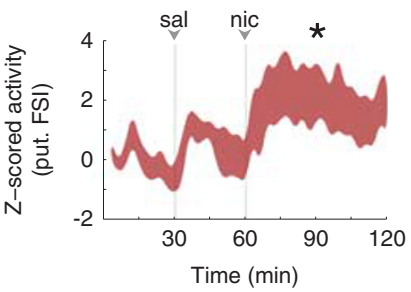

Figure 3 Nicotine altered medial prefrontal cortex (mPFC) unit activity upon daily exposures. (a) Overview of mPFC unit activity throughout nicotine sessions. The rows in each image display unit firing rates (Z-scores; I min bins) from multiple rats. Units were sorted according to mean post-nicotine Z-scores. (b) Proportions of units that were excited (red), inhibited (blue) or unaffected (green) by nicotine exposure, according to $t$-test comparisons between baseline and post-drug firing rates. A greater proportion of units was inhibited on the first session compared with subsequent sessions, as confirmed by a $\chi^{2}$ test. (c) Top and bottom panels compare population activities and their absolute values, respectively, either between days I and 3 (left) or between days I and 5 (right). Shaded curves represent \pm SEM, and Tukey's significant effects are indicated ( 3 min bins). (d) The scatterplot on the left contains all mPFC units from all sessions ( $n=200)$, distributed according to baseline firing rates and spike widths (time between action potential valley and hyperpolarization peak). The red rectangle represents the criterion to identify putative fast-spiking interneuron (FSI) from the mPFC ( $>10 \mathrm{~Hz}$ firing rate, $<0.3$ ms spike width (Homayoun and Moghaddam, 2007); $n=5$ ). Right panel shows the activity of these units, which were excluded from the analyses described in panels (a-c). Nicotine increased the activity of putative FSI, according to one-way ANOVA (asterisk).

Table 2 Proportion of Single Unit Categories and their Baseline Firing Rates $(\mathrm{Hz})$

$$
\text { Day I }(n=70)
$$

\section{Proportion (\%)}

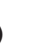

Basal firing

\begin{tabular}{lll}
\hline Excited & 24.29 & $2.16 \pm 0.38$ \\
Unchanged & 18.57 & $2.48 \pm 0.73$ \\
Inhibited & 57.14 & $2.69 \pm 0.50$ \\
\hline
\end{tabular}

Basal firing rates listed as mean \pm SEM. The number of units $(n)$ isolated from 15 rats is depicted for each day.

Day $3(n=64)$

\begin{tabular}{ccccc}
\hline Proportion (\%) & Basal firing & & Proportion (\%) & Basal firing \\
\hline 32.81 & $2.80 \pm 0.45$ & & 29.51 & $2.47 \pm 0.36$ \\
34.38 & $2.78 \pm 0.40$ & & 34.43 & $2.10 \pm 0.29$ \\
32.81 & $2.39 \pm 0.53$ & 36.06 & $2.39 \pm 0.34$
\end{tabular}

difference in baseline attentional accuracy between nicotine and saline groups $\left(\mathrm{t}_{(1,15)}=0.85, p=0.4\right)$. As expected, we observed an effect of cue length on attention $\left(\mathrm{F}_{(2,30)}=19.94\right.$, $p<0.001)$, indicating that rats were more accurate on trials with longer cues. Nicotine did not affect attention during long cue trials across treatment days $\left(\mathrm{F}_{(1,15)}=0.892\right.$, $p=0.360)$, but improved attention during the more difficult mid- and short-length cues (mid: $\mathrm{F}_{(1,15)}=5.234, p=0.04$; short: $\left.F_{(1,15)}=5.495, p=0.03\right)$. Nicotine did not significantly improve performance on non-cued trials, although there was a trend toward improvement $\left(\mathrm{F}_{(1,15)}=2.15, p=0.16\right)$. When all cue lengths were merged, nicotine increased accuracy compared with saline $\left(\mathrm{F}_{(1,15)}=6.70, p=0.02\right.$; Figure $\left.4 \mathrm{a}\right)$. In addition, there was a significant drug $v s$ session interaction $\left(\mathrm{F}_{(4,60)}=4.5, p=0.003\right)$. There was no nicotine effect on day $1\left(t_{(15)}=0.74, p=0.5\right)$, near significant attentional enhancement by nicotine on days $2-3\left(\mathrm{~d} 2: t_{(15)}=2.39, p=0.03\right.$; d3: $\left.t_{(15)}=1.99, p=0.06\right)$, and significant increases in accuracy on days $4-5\left(\mathrm{~d} 4: t_{(15)}=3.09, p=0.007 ; \mathrm{d} 5: t_{(15)}=2.93, p=0.01\right)$. Thus, nicotine did not influence attention upon first exposure, but subsequent daily treatments improved attention. We repeated this test after the 9-day nicotine washout, and found a near significant difference between groups $\left(t_{(15)}=2.023, P=0.06\right.$; Figure $\left.4 \mathrm{a}\right)$. Thus, post-washout nicotine improved attention similarly to days $2-3$. Importantly, the improved attention throughout nicotine exposure 
a
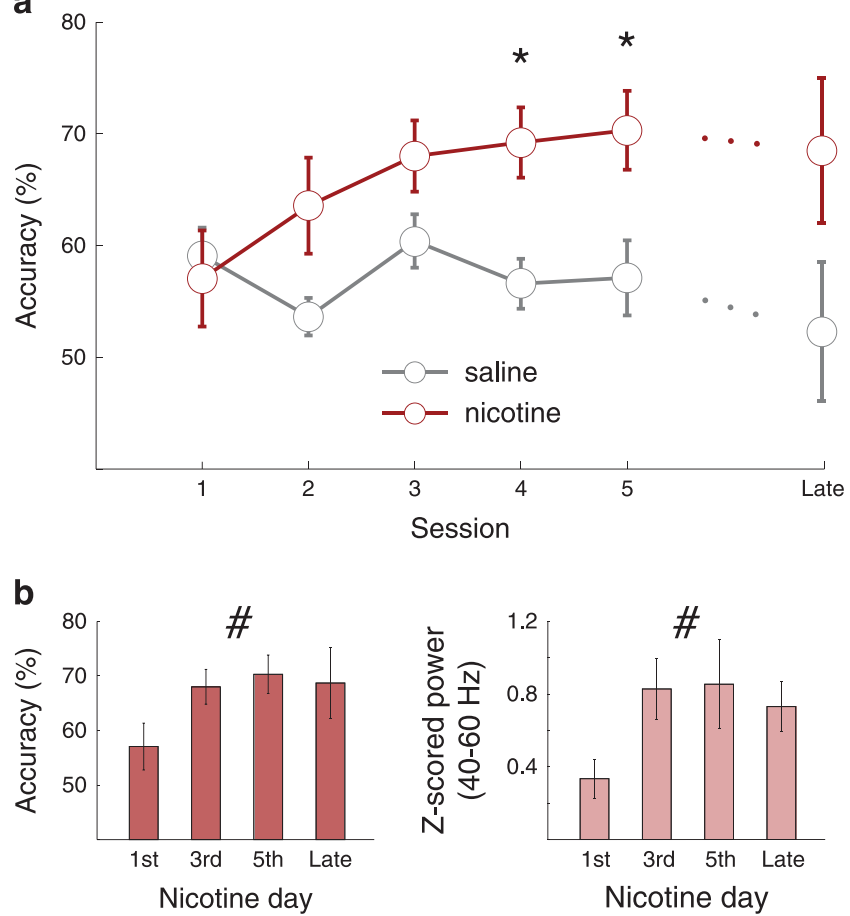

Figure 4 Nicotine improved visual attention upon daily exposures, similar to the electrophysiological alterations. (a) Visual attention accuracy throughout the daily regimen of $0.2 \mathrm{mg} / \mathrm{kg}$ nicotine, as measured by the percentage of correct cued responses. Asterisks indicate t-test significant nicotine vs saline differences. (b) Data from the nicotine-exposed rats highlighting that the visual attention improvement (left) paralleled the low gamma potentiation (right), also shown in Figure 2. \# indicates withinsession effects.

$\left(\mathrm{F}_{(4,28)}=4.14, p=0.009\right)$ followed a similar session-specific pattern to the increase in $\mathrm{mPFC}$ low gamma power $\left(F_{(2,20)}=3.51, p=0.05\right.$; Figure $\left.4 b\right)$.

Nicotine did not affect premature responding on any sessions (sessions $1-5: \mathrm{F}_{(1,15)}=0.09, p=0.767$; late session: $\left.t_{(15)}=1.55, p=0.14\right)$, nor did nicotine affect perseverative errors on sessions $1-5\left(\mathrm{~F}_{(1,15)}=0.02, p=0.88\right)$ or the late session $\left(t_{(15)}=1.05, p=0.31\right)$. In addition, there were no differences in latency to perform a response across the five sessions $\left(\mathrm{F}_{(1,15)}=0.31, p=0.6\right)$ or during the late session $\left(t t_{(15)}=0.31, p=0.8\right)$. There also was no effect of nicotine on total omitted trials across all sessions $\left(\mathrm{F}_{(1,15)}=2.64, p=0.13\right)$, although the first exposure increased omissions $\left(t_{(15)}=2.86\right.$, $p=0.01)$. In general, effects of nicotine on cognition were specific to attention.

\section{DISCUSSION}

Daily nicotine exposure led to strengthening of gamma LFP oscillations in mPFC in correlation with behavioral sensitization. This pattern of plasticity was specific to the gamma band as lower frequency LFP oscillations (theta and beta) were suppressed consistently across daily nicotine treatments. Expression of stronger gamma oscillations persisted after a washout period and was associated with a shift in inhibitory-excitatory balance in single-unit activity. To provide a functional interpretation for these LFP data, visual attention was tested in a different group of subjects during a similar 5-day nicotine regimen. Nicotine improved attention only after multiple daily treatments, mirroring the pattern of gamma modulation. This suggests that an oscillatory plasticity in the gamma range in the $\mathrm{mPFC}$ is relevant to the emergence of behavioral sensitization and cognitive enhancement by nicotine.

\section{Relevance to Previous Literature}

Nicotine causes synaptic plasticity in PFC slices (Lambe et al, 2003; Mansvelder et al, 2009), increases CREB phosphorylation, and causes an increase in PFC dendritic spines (Brunzell et al, 2003; Gonzalez et al, 2005). Here, we demonstrate that these alterations manifest in behaving animals as increased PFC gamma power, decreased theta/ beta power, and a balanced activation/suppression of neuronal firing rate. More importantly, we find that gamma modulation is exquisitely sensitive to repeated exposure to nicotine and may, therefore, be a mode of plasticity associated with nicotine.

In a separate cohort of animals, we observed that the same pattern of nicotine injection that led to the emergence of a stronger gamma also improved accuracy during a visual attention task. This is consistent with previous studies showing that extended exposure to nicotine and nicotinic agonists enhances attention (Amitai and Markou, 2008; Levin et al, 2006; McGaughy et al, 1999). In particular, Pattij et al (2007) and Semenova et al (2007) observed that multiple days of repeated nicotine exposure were necessary to improve attention in rats.

The area of the mPFC we targeted here is often designated as the prelimbic PFC (PL). This region demonstrates sustained firing during visual attention (Totah et al, 2009). In addition, synchrony between the $\mathrm{PL}$ and anterior cingulate cortex (ACC), the brain region classically associated with attention, predicts attentional performance (Totah et al, 2013). It is proposed that PL is involved with linking cues with specific responses during attention, whereas ACC links cues with outcomes (Shidara and Richmond, 2002; Totah et al, 2009). Future studies assessing the functional interaction between PL and ACC during nicotine exposure and attention could shed further light on how nicotine modulates attention.

\section{Interpretation of Changes in Single Unit Activity}

We observed a heterogeneous population response after nicotine treatment. This was expected given that other psychoactive drugs, despite targeting specific receptors, also produce mixed responses in the PFC (Wood et al, 2012). Despite this variability, there was a net inhibition following the first nicotine administration, which shifted toward a balance of inhibition and excitation across all subsequent sessions. The first exposure to nicotine is typically aversive in adult rats (Wilmouth and Spear, 2004). The overall mPFC inhibition observed on day 1 may be related to this aversion, as an anxiogenic state has been associated with mPFC inhibition (Park et al, 2016). Accordingly, in the attentional task, rats omitted a greater number of trials on day 1 of nicotine, which also may be a result of increased anxiety. With ongoing nicotine exposure, tolerance for nicotine aversion is increased (Fowler and Kenny, 2014), which 
may be reflected by the shift toward excitatory-inhibitory balance. Repeated nicotine exposure has been shown to cause an upregulation in $\beta 2$-containing nicotinic receptors in mPFC, primarily located on pyramidal neurons (Poorthuis and Mansvelder, 2013). This increase in excitatory input may underlie the shift from inhibition to a more balanced pattern of ensemble activity. Future studies will be required to evaluate this or other mechanisms.

\section{Relevance of Gamma Oscillations to Cognition}

Several cognitive functions, including attention and working memory, are associated with enhanced gamma oscillations (Fries et al, 2001; Gregoriou et al, 2015; Lundqvist et al, 2016). The role of gamma in cognition may result from regulation of millisecond-range synchrony between neuronal ensembles, dynamically establishing information channels between brain structures (Bartos et al, 2007; Benchenane et al, 2011). In support of this hypothesis, gamma oscillations in primate PFC and visual cortex are coupled during visual attention, suggesting that PFC gamma entrains sensory regions to attend to appropriate stimuli (Gregoriou et al, 2009). Our data support this connection between gamma oscillations and cognitive performance, as nicotine-induced physiological and behavioral changes followed the same timeline.

Several recent studies have revealed a causal relationship between PFC gamma, top-down cognition, and GABAergic FSI; namely, stimulating FSI enhances visual attention (Kim et al, 2016), whereas inhibiting FSI suppresses gamma and impairs cognitive flexibility (Cho et al, 2015). GABA neurons in the PFC express nicotinic receptors (Aracri et al, 2010), receive cholinergic innervations (Henny and Jones, 2008), and entrain pyramidal neuron firing into gamma frequencies (Sohal et al, 2009). Thus, nicotinic receptors are ideally situated to regulate PFC gamma oscillations. We observed that daily nicotine exposure modulates $\mathrm{MPFC}$ gamma power, although this control over gamma required multiple treatments to manifest. This indicates that priming of nicotinic receptor subtypes (eg, long-term dynamics of receptor desensitization or upregulation due to daily nicotine) may be necessary before gamma oscillations can be affected. Importantly, attentional performance also was enhanced after a comparable amount of exposure. This parallel pattern of modulation suggests that increased gamma power must reach a threshold for nicotine to improve attention.

\section{Nicotinic Receptors as a Target for Cognitive Enhancement in Psychopathology}

The cognitive enhancing effects of nicotine have been well established. Nicotine improves attention, working memory, and memory consolidation in both animals and humans (Levin et al, 2006; Rushforth et al, 2010; Semenova et al, 2007; Warburton, 1992). Many of these cognitive functions are also impaired in schizophrenia, and this impairment is notoriously difficult to treat with antipsychotic medication (Green, 1996; Kahn and Keefe, 2013; Olincy and Freedman, 2012). Some patients have reported that nicotine improves their cognitive abilities, and smoking is highly comorbid with schizophrenia, suggesting that it may be a method of 'selfmedication' (Dalack et al, 1998). Furthermore, decreased nicotinic receptor activity has been observed in PFC of patients when compared with controls (Breese et al, 2000). Collectively, these data suggest that reduced nicotinic receptor activity may contribute to cognitive deficits of schizophrenia (Parikh et al, 2016). This has facilitated development of nicotinic receptor agonists as a treatment for schizophrenia, particularly targeting the alpha-7 receptor (Olincy and Freedman, 2012). Unfortunately, the outcomes of these treatments in normalizing pathological cognition have been mixed. The experimental alpha-7 partial agonist encenicline showed efficacy as a cognitive enhancer in schizophrenics in Phase 2 trials, but Phase 3 trials have been less successful (Rogers, 2016). Our data suggest that (1) PFC gamma oscillations and improvements in attention arise in parallel and (2) these effects are strengthened after multiple treatments of a consistent dose of nicotine. Therefore, the effectiveness of nicotinic drugs may be related to maintaining a specific regimen and dose of administration. Further studies can be used to determine the specific threshold dose and schedule required to enhance gamma power and attention. It may be necessary to titrate the dose and schedule of a drug on a caseby-case basis to modulate cortical gamma oscillations, which can be measured non-invasively in humans using EEG or MEG. Disruption of cognition in schizophrenia is characterized by abnormal production and synchronization of gamma rhythms in PFC (Sohal et al, 2009; Uhlhaas and Singer, 2013); measuring how nicotine increases or normalizes aberrant gamma may be an effective method of assessing treatment potential in individualized medicine.

\section{Nicotine Withdrawal Increases Nicotine Control Over High Gamma Oscillations}

Behavior and neuronal activity in response to nicotine was also measured after an extended withdrawal period. Previous studies have shown that withdrawal enhances the behavioral and synaptic response to drugs of abuse, a phenomenon known as sensitization (Robinson and Becker, 1986; Vezina et al, 2007). Sensitization is associated with drug craving, increasing the risk of relapse (Robinson and Berridge, 1993). Here, we observed that post-withdrawal nicotine increases both low and high gamma oscillations in MPFC, with a much greater effect on high gamma than during the initial nicotine regimen. Because increased high gamma power in $\mathrm{mPFC}$ was most evident after withdrawal, this oscillatory profile may serve as a biomarker of nicotine sensitivity and vulnerability to addiction. The dissociation between low and high gamma revealed by the washout period could also be hypothesized to influence attention, as differential involvements of these two bands in cognition have been reported (Catanese et al, 2016; Chaumon et al, 2009; van der Meer and Redish, 2009). However, attention performance showed no clear distinctions between the post-washout session and the second half of the daily dosing period. Thus, observing low and high gamma may be an effective way of distinguishing nicotine's cognitive enhancing effects from its addiction-relevant rewarding effects.

\section{CONCLUSION}

We show that daily exposure to nicotine enhances its effect on gamma oscillatory activity and its procognitive properties. 
In the context of the clinical use of nicotine and related agonists for treating cognitive deficits, we propose that (1) repeat dosing may be critical for clinical efficacy and (2) oscillatory plasticity in the gamma range may provide a biomarker for assessing this efficacy.

\section{FUNDING AND DISCLOSURE}

This work was supported by São Paulo Research Foundation, Brazil (FAPESP; fellowships 2012/21387-8 and 2012/061234) for investigator LSBJ, R01MH084906 (BM), and a pilot fund from the Center for Evaluation of Nicotine in Cigarettes (NWS). The authors declare no conflict of interest.

\section{ACKNOWLEDGMENTS}

We thank Corey Patterson and Karen Nieri for technical support, and Drs Jesse Wood, Yunbok Kim, Junchol Park, Alberto Del Arco, and Adria Martig for helpful discussions and valuable input on data analysis and experimental design.

\section{REFERENCES}

Amitai N, Markou A (2008). Chronic nicotine improves cognitive performance in a test of attention but does not attenuate cognitive disruption induced by repeated phencyclidine administration. Psychopharmacology 202: 275-286.

Aracri P, Consonni S, Morini R, Perrella M, Rodighiero S, Amadeo A et al (2010). Tonic modulation of GABA release by nicotinic acetylcholine receptors in layer $\mathrm{V}$ of the murine prefrontal cortex. Cereb Cortex 20: 1539-1555.

Bartos M, Vida I, Jonas P (2007). Synaptic mechanisms of synchronized gamma oscillations in inhibitory interneuron networks. Nat Rev Neurosci 8: 45-56.

Benchenane K, Tiesinga PH, Battaglia FP (2011). Oscillations in the prefrontal cortex: a gateway to memory and attention. Curr Opin Neurobiol 21: 475-485.

Breese CR, Lee MJ, Adams CE, Sullivan B, Logel J, Gillen KM et al (2000). Abnormal regulation of high affinity nicotinic receptors in subjects with schizophrenia. Neuropsychopharmacology 23: 351-364.

Brunzell DH, Russell DS, Picciotto MR (2003). In vivo nicotine treatment regulates mesocorticolimbic CREB and ERK signaling in C57Bl/6J mice. J Neurochem 84: 1431-1441.

Catanese J, Carmichael JE, van der Meer MA (2016). Low- and high-gamma oscillations deviate in opposite directions from zerophase synchrony in the limbic corticostriatal loop. J Neurophysiol 116: 5-17.

Chang PY, Lo PC, Chang HC, Hsueh KC, Tsai YW (2016). Comparative effectiveness of smoking cessation medications: a national prospective cohort from Taiwan. PLOS ONE 11: e0166992.

Chaumon M, Schwartz D, Tallon-Baudry C (2009). Unconscious learning versus visual perception: dissociable roles for gamma oscillations revealed in MEG. J Cogn Neurosci 21: 2287-2299.

Cho KKA, Hoch R, Lee AT, Patel T, Rubenstein JLR, Sohal VS (2015). Gamma rhythms link prefrontal interneuron dysfunction with cognitive inflexibility in Dlx5/6(+/-) mice. Neuron 85: 1332-1343.

Dalack GW, Healy DJ, Meador-Woodruff JH (1998). Nicotine dependence in schizophrenia: clinical phenomena and laboratory findings. Am J Psychiatry 155: 1490-1501.

De Biasi M, Dani JA (2011). Reward, addiction, withdrawal to nicotine. Annu Rev Neurosci 34: 105-130.

DiFranza JR, Wellman RJ (2007). Sensitization to nicotine: how the animal literature might inform future human research. NicotineTob Res 9: 9-20.
Ferger B, Kuschinsky K (1994). Activation of dopamine D1 receptors or alpha 1 adrenoceptors is not involved in the EEG effect of nicotine in rats. Naunyn-Schmiedeberg's Archiv Pharmacol 350: 346-351.

Ferrarelli F, Massimini M, Peterson MJ, Riedner BA, Lazar M, Murphy MJ et al (2008). Reduced evoked gamma oscillations in the frontal cortex in schizophrenia patients: a TMS/EEG study. Am J Psychiatry 165: 996-1005.

Fowler CD, Kenny PJ (2014). Nicotine aversion: neurobiological mechanisms and relevance to tobacco dependence vulnerability. Neuropharmacology 76 (Pt B): 533-544.

Freedman R (2014). Alpha7-nicotinic acetylcholine receptor agonists for cognitive enhancement in schizophrenia. Annu Rev Med 65: 245-261.

Fries P, Reynolds JH, Rorie AE, Desimone R (2001). Modulation of oscillatory neuronal synchronization by selective visual attention. Science 291: 1560-1563.

Gonzalez CL, Gharbawie OA, Whishaw IQ, Kolb B (2005). Nicotine stimulates dendritic arborization in motor cortex and improves concurrent motor skill but impairs subsequent motor learning. Synapse 55: 183-191.

Green MF (1996). What are the functional consequences of neurocognitive deficits in schizophrenia? Am J Psychiatry 153: 321-330.

Gregoriou GG, Gotts SJ, Zhou H, Desimone R (2009). Highfrequency, long-range coupling between prefrontal and visual cortex during attention. Science 324: 1207-1210.

Gregoriou GG, Paneri S, Sapountzis P (2015). Oscillatory synchrony as a mechanism of attentional processing. Brain Res 1626: 165-182.

Henny P, Jones BE (2008). Projections from basal forebrain to prefrontal cortex comprise cholinergic, GABAergic and glutamatergic inputs to pyramidal cells or interneurons. Eur $J$ Neurosci 27: 654-670.

Homayoun H, Moghaddam B (2007). NMDA receptor hypofunction produces opposite effects on prefrontal cortex interneurons and pyramidal neurons. J Neurosci 27: 11496-11500.

Kahn RS, Keefe RE (2013). Schizophrenia is a cognitive illness: time for a change in focus. JAMA Psychiatry 70: 1107-1112.

Kim H, Ahrlund-Richter S, Wang X, Deisseroth K, Carlen M (2016). Prefrontal parvalbumin neurons in control of attention. Cell 164: 208-218.

Lambe EK, Picciotto MR, Aghajanian GK (2003). Nicotine induces glutamate release from thalamocortical terminals in prefrontal cortex. Neuropsychopharmacology 28: 216-225.

Levin ED, McClernon FJ, Rezvani AH (2006). Nicotinic effects on cognitive function: behavioral characterization, pharmacological specification, and anatomic localization. Psychopharmacology (Berl) 184: 523-539.

Lundqvist M, Rose J, Herman P, Brincat SL, Buschman TJ, Miller EK (2016). Gamma and beta bursts underlie working memory. Neuron 90: 152-164.

Mansvelder HD, Mertz M, Role LW (2009). Nicotinic modulation of synaptic transmission and plasticity in cortico-limbic circuits. Semin Cell Dev Biol 20: 432-440.

Markou A (2008). Review. Neurobiology of nicotine dependence. Philos Trans R Soc Lond B Biol Sci 363: 3159-3168.

McGaughy J, Decker MW, Sarter M (1999). Enhancement of sustained attention performance by the nicotinic acetylcholine receptor agonist ABT-418 in intact but not basal forebrainlesioned rats. Psychopharmacology (Berl) 144: 175-182.

McGaughy J, Sarter M (1995). Behavioral vigilance in rats: task validation and effects of age, amphetamine, and benzodiazepine receptor ligands. Psychopharmacology (Berl) 117: 340-357.

Mitra P, Bokil H (2008). Observed Brain Dynamics. Oxford University Press: New York.

Olincy A, Freedman R (2012). Nicotinic mechanisms in the treatment of psychotic disorders: a focus on the alpha7 nicotinic receptor. Handb Exp Pharmacol 213: 211-232. 
Parikh V, Kutlu MG, Gould TJ (2016). nAChR dysfunction as a common substrate for schizophrenia and comorbid nicotine addiction: current trends and perspectives. Schizophr Res 171: $1-15$.

Park J, Wood J, Bondi C, Del Arco A, Moghaddam B (2016). Anxiety evokes hypofrontality and disrupts rule-relevant encoding by dorsomedial prefrontal cortex neurons. J Neurosci 36: 3322-3335.

Pattij T, Janssen MC, Loos M, Smit AB, Schoffelmeer AN, van Gaalen MM (2007). Strain specificity and cholinergic modulation of visuospatial attention in three inbred mouse strains. Genes Brain Behav 6: 579-587.

Picciotto MR (1998). Common aspects of the action of nicotine and other drugs of abuse. Drug Alcohol Depend 51: 165-172.

Poorthuis RB, Mansvelder HD (2013). Nicotinic acetylcholine receptors controlling attention: behavior, circuits and sensitivity to disruption by nicotine. Biochem Pharmacol 86: 1089-1098.

Robinson TE, Becker JB (1986). Enduring changes in brain and behavior produced by chronic amphetamine administration: a review and evaluation of animal models of amphetamine psychosis. Brain Res 396: 157-198.

Robinson TE, Berridge KC (1993). The neural basis of drug craving: an incentive-sensitization theory of drug-addiction. Brain Res Brain Res Rev 18: 247-291.

Rogers MB (2016). Encenicline misses endpoints in two phase 3 schizophrenia trials. Schizophr Res Forum. Available at http://www.schizophreniaforum.org/news/encenicline-misses-end points-two-phase-3-schizophrenia-trials.

Rushforth SL, Allison C, Wonnacott S, Shoaib M (2010). Subtypeselective nicotinic agonists enhance olfactory working memory in normal rats: a novel use of the odour span task. Neurosci Lett 471: $114-118$.

Semenova S, Stolerman IP, Markou A (2007). Chronic nicotine administration improves attention while nicotine withdrawal induces performance deficits in the 5-choice serial reaction time task in rats. Pharmacol Biochem Behav 87: 360-368.

Shidara M, Richmond BJ (2002). Anterior cingulate: single neuronal signals related to degree of reward expectancy. Science 296: $1709-1711$.

Sohal VS, Zhang F, Yizhar O, Deisseroth K (2009). Parvalbumin neurons and gamma rhythms enhance cortical circuit performance. Nature 459: 698-702.
Totah NK, Jackson ME, Moghaddam B (2013). Preparatory attention relies on dynamic interactions between prelimbic cortex and anterior cingulate cortex. Cereb Cortex 23: 729-738.

Totah NK, Kim YB, Homayoun H, Moghaddam B (2009). Anterior cingulate neurons represent errors and preparatory attention within the same behavioral sequence. J Neurosci 29: 6418-6426.

Uhlhaas PJ, Singer W (2013). High-frequency oscillations and the neurobiology of schizophrenia. Dialogues Clin Neurosci 15: 301-313.

van der Meer MA, Redish AD (2009). Low and high gamma oscillations in rat ventral striatum have distinct relationships to behavior, reward, and spiking activity on a learned spatial decision task. Front Integr Neurosci 3: 9.

Vezina P, McGehee DS, Green WN (2007). Exposure to nicotine and sensitization of nicotine-induced behaviors. Progr Neuropsychopharmacol Biol Psychiatry 31: 1625-1638.

Wang Y, Wang Z, Wang J, Henderson Z, Wang X, Zhang X et al (2015). The modulation of nicotinic acetylcholine receptors on the neuronal network oscillations in rat hippocampal CA3 area. Sci Rep 5: 9493.

Warburton DM (1992). Nicotine as a cognitive enhancer. Progr Neuro-psychopharmacol Biol Psychiatry 16: 181-191.

Wilmouth CE, Spear LP (2004). Adolescent and adult rats' aversion to flavors previously paired with nicotine. Ann NY Acad Sci 1021: 462-464.

Wood J, Kim Y, Moghaddam B (2012). Disruption of prefrontal cortex large scale neuronal activity by different classes of psychotomimetic drugs. J Neurosci 32: 3022-3031.

(i) (-) $\odot$ This work is licensed under a Creative Commons Attribution-NonCommercial-NoDerivs $\quad 4.0$ International License. The images or other third party material in this article are included in the article's Creative Commons license, unless indicated otherwise in the credit line; if the material is not included under the Creative Commons license, users will need to obtain permission from the license holder to reproduce the material. To view a copy of this license, visit http://creativecommons.org/licenses/by-nc-nd/4.0/

(C) The Author(s) 2017 